M. Stöhr, K. P. Geigle, R. Hadef, I. Boxx, C. D. Carter, M. Grader and P. Gerlinger, Time-resolved study of transient soot formation in an aero-engine model combustor at elevated pressure, Proceedings of the Combustion Institute 37 (2019) 5421-5428.

The original publication is available at www.elsevier.com

https://doi.org/10.1016/j.proci.2018.05.122 


\title{
Time-resolved study of transient soot formation in an aero-engine model combustor at elevated pressure
}

\author{
M. Stöhra, ${ }^{\mathrm{a}, *}$, K. P. Geigle ${ }^{\mathrm{a}}$, R. Hadef ${ }^{\mathrm{b}}$, I. Boxx ${ }^{\mathrm{a}}$, C. D. Carter $^{\mathrm{c}}$, M. Grader ${ }^{\mathrm{a}}$, P. Gerlinger ${ }^{\mathrm{a}}$ \\ ${ }^{a}$ German Aerospace Center (DLR), Institute of Combustion Technology, Pfaffenwaldring 38-40, 70569 Stuttgart, Germany \\ ${ }^{b}$ Université Larbi Ben M'Hidi, Institut de Génie Mécanique, 04000 Oum El Bouaghi, Algeria \\ ${ }^{c}$ Air Force Research Laboratory, Wright-Patterson Air Force Base, Dayton, OH 45433, USA
}

\begin{abstract}
The mechanisms of transient formation and oxidation of soot in an aero-engine model combustor at elevated pressure are studied for the first time using a combination of high-speed simultaneous stereo-PIV and OH-PLIF and results from a recent detailed LES. A combined analysis of experiment and LES shows that the highly transient and intermittent evolution of soot in this combustor is governed by an unsteady interplay of distinct pockets of burned gas in the inner recirculation zone (IRZ) with either relatively rich or relatively lean composition. The former originate from reaction of fuel-rich unburned gas, whereas the latter result from additional admixture of secondary air further downstream. The analysis further enables distinction and localization of premixed and diffusion-type flame fronts within the flame zone. The time-resolved complementary measurements of velocity field and flame structure allow accurate tracking of both the burned gas pockets and soot filaments. It is seen that soot generally forms in the rich pockets if their residence time in the IRZ is sufficient, whereas oxidation occurs in the lean zones carrying OH. Correlating the dynamics of flow field and soot indicates that the intermittency of soot is driven by an intermittent flow of lean burned gas into the IRZ that affects the residence time of rich pockets. The results suggest that the formation of soot might be further reduced by a proper adjustment of secondary air injection aiming at a sufficient and more constant recirculation of lean burned gas. A remarkably good agreement of measured and simulated instantaneous flame structures is observed, indicating that flow field and gas-phase reactions are well predicted by the LES. The experimental insights into the transient mechanisms of soot formation and oxidation, on the other hand, may provide useful input for LES soot models where deviations from measurements are generally larger.
\end{abstract}

Keywords:

Soot formation, Aero-engine combustor, Turbulent swirl flame, Highspeed laser measurements, Large eddy simulation

${ }^{*}$ Corresponding author:

Email address: michael.stoehr@dlr.de (M. Stöhr) 


\section{Introduction}

The harmful effects of combustion-generated soot particles on human health and environment, and associated stringent emission regulations necessitate the improvement of aero-engine combustor emission characteristics [1]. While the fundamental understanding of soot formation has evolved considerably during the last decades [2], accurate predictive modeling of soot is still a major challenge in realistic combustors featuring complex turbulent swirl flows, where intricate unsteady fuel-air mixing, a wide range of time scales between high-speed jets and low-velocity recirculation zones, and possible hydrodynamic and acoustic instabilities affect soot formation and oxidation [3, 4].

Detailed experimental studies are essential for improving the understanding of soot evolution and providing accurate data for model validation. The availability of accurate experimental data for technically relevant combustors under well-defined conditions yet still remains limited. Recently our group has reported measurements of velocity field, temperature and soot concentration as well as visualization of $\mathrm{OH}$ and $\mathrm{PAH}$ for an aero-engine model combustor at elevated presssure $[3,5,6]$. The data is available on request and has since been used for improvement and validation of large eddy simulation (LES) calculations [7-9].

An advanced understanding of the highly transient and history-dependent soot formation processes, however, was limited by the low repetition rates of the reported measurements. In recent years the use of timeresolved measurements has considerably enhanced the understanding of soot formation in turbulent jet flames [10-13], thus demonstrating the potential of this approach. The present work aims at elucidating the complex mechanisms of soot formation in a technically relevant aero-engine model combustor for the first time using high-speed laser-based techniques. Since the flame exhibits a complex structure with different thermochemical states and the number of measurable quantities is limited, the analysis is supported by results from a recent detailed LES of the same flame by Grader et al. [7].

\section{Methods}

\subsection{Combustor and operating condition}

The study employs a single-injector swirl combustor (shown in Fig. 1) that is representative of one sector of an annular aero-engine combustor. The injector comprises two concentric swirl nozzles for air with outer diameters of $d=12.3$ and $19.8 \mathrm{~mm}$. The two air flows are first fed into separate plenums and then pass radial swirlers composed of eight channels for the central nozzle and 12 channels for the outer nozzle. In order to avoid additional complexities from liquid fuel atomization and evaporation, the burner is operated with gaseous fuel, which is injected without swirl through 60 thin channels $\left(0.5 \times 0.4 \mathrm{~mm}^{2}\right)$ located on a ring between the air nozzles. The exit planes of the air and fuel nozzles are mounted flush with the base plate of the combustion chamber that is defined as $y=0 \mathrm{~mm}$. The chamber has a square cross-section of $68 \times 68 \mathrm{~mm}^{2}$, a height of $120 \mathrm{~mm}$ and a cylindrical exhaust gas exit of $d=40$ $\mathrm{mm}$. The four side walls are made of quartz windows with a thickness of $3 \mathrm{~mm}$, which provide extensive optical access to the flame. The windows are held by 4 metal posts in the corners of the chamber that each have a duct of $d=5 \mathrm{~mm}$ at $y=80 \mathrm{~mm}$ for injection of secondary air. The latter represents the dilution or quench air used in aero-engine burners. The combustor is mounted in a water-cooled steel pressure housing with large optical access of $60 \times 120 \mathrm{~mm}^{2}$ from four sides. Further details of the combustor and test rig are provided in Refs. [3, 5].

For the present study the combustor was operated at a pressure of 3 bar with ethylene and air, both injected at ambient temperature. The thermal power and equivalence ratio with respect to the main injector are $P=32.2$ $\mathrm{kW}$ and $\phi=1.2$, respectively. In addition, secondary air is injected through the ducts at $y=80 \mathrm{~mm}$, which results in global values of $P$ and $\phi$ of $38.6 \mathrm{~kW}$ and 0.86 , respectively. As seen in Fig. 1, the flame base is located $\approx 10 \mathrm{~mm}$ above the nozzle exit. Therefore a certain degree of fuel-air mixing takes place before reaction, and thus the flame is considered as partially premixed. For the same flame, various laser-based measurements with low repetition rates are presented in Refs. $[3,5,6]$.

\subsection{Measuring techniques}

Time-resolved fields of velocity and flame structure were measured using combined stereoscopic particle image velocimetry (stereo-PIV) and planar laserinduced fluorescence (PLIF) of $\mathrm{OH}$ operated at $\mathrm{kHz}$ repetition rates. The stereo-PIV setup consisted of a dual-cavity, diode-pumped, solid state laser (Edgewave, IS200-2-LD, $9 \mathrm{~mJ} /$ pulse at $532 \mathrm{~nm}$ ) and a pair of CMOS cameras (LaVision HSS8) mounted at a vertical angle on opposite sides of the combustor. The laser beam was formed into a sheet with a height of $40 \mathrm{~mm}$ using a set of cylindrical lenses. The air flows were seeded with $\mathrm{TiO}_{2}$ tracer particles $(d \approx 1 \mu \mathrm{m})$. Particle image pairs with a resolution of $512 \times 640$ pixels and a separation time of $10 \mu \mathrm{s}$ were acquired at a rate of $9.3 \mathrm{kHz}$. Image mapping, calibration, particle image cross-correlation and 


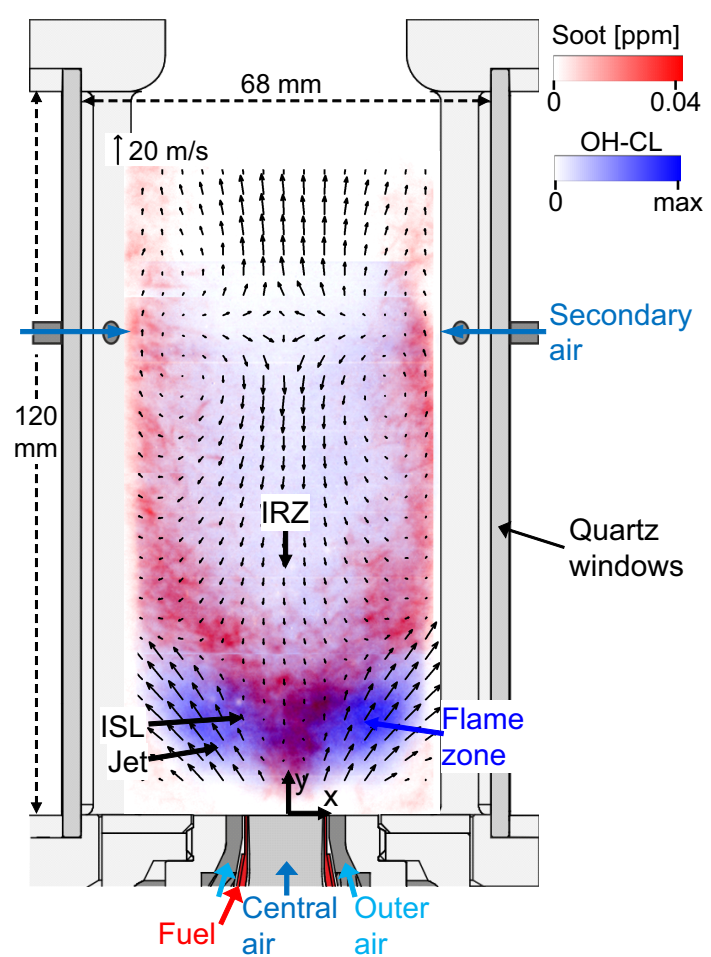

Figure 1: Schematic of combustion chamber with plots of average velocity field, soot concentration and $\mathrm{OH}-\mathrm{CL}$ measurements.

uncertainty quantification were completed using a commercial software (LaVision DaVis 8.2). Apart from Mie scattering from tracer particles, strong Rayleigh scattering from soot particles was encountered as shown below in Sect. 4. Since the PIV cross-correlation with final interrogation window sizes of $32 \times 32$ or fewer pixels failed at locations where large soot filaments are present, a relatively large window size of $48 \times 48$ pixels, corresponding to a spatial resolution of $4 \mathrm{~mm}$ was chosen. Using a window overlap of $50 \%$, the vector spacing was $2 \mathrm{~mm}$. The uncertainty of instantaneous velocities based on the correlation statistics approach by Wieneke [14] was determined as $\approx 0.5 \mathrm{~m} / \mathrm{s}$ for the in-plane components and $\approx 1 \mathrm{~m} / \mathrm{s}$ for the out-of-plane component

The OH-PLIF system used a frequency-doubled dye laser (Sirah Cobra-Stretch HRR) driven by a frequencydoubled, diode-pumped solid state laser (Edgewave IS$811 \mathrm{E})$. The laser was tuned to the overlapping $\mathrm{Q}_{2}(8)$ and $\mathrm{Q}_{1}(9)$ lines of the A-X $(1,0)$ band of $\mathrm{OH}$ at $283.9 \mathrm{~nm}$. The system was operated at a rate of $3.1 \mathrm{kHz}$ where a pulse energy of $226 \mu \mathrm{J}$ was obtained. The beam was formed into a sheet with a height of $\approx 40 \mathrm{~mm}$ using fused silica cylindrical lenses and overlapped with the PIV sheet using a pair of dichroic mirrors. The fluorescence signal was acquired using a CMOS camera (LaVision HSS6) with an external two-stage intensifier (LaVision HS-IRO, $100 \mathrm{~ns}$ integration time), a $f=100 \mathrm{~mm}, f / 2.8$ UV lens (Cerco) and a bandpass interference filter. The PLIF recordings were timed between the two PIV laser pulses of every third PIV recording. A series of 24576 PIV and 8192 PLIF measurements was recorded, corresponding to a duration of 2.64 seconds.

\subsection{Numerical simulation}

In order to support interpretation of the complex spatial structures seen in the OH-PLIF measurements, results from a recent detailed LES of the same flame by Grader et al. [7] are employed. The LES uses the DLR finite-volume solver THETA that is optimized for combustion simulations on unstructured grids [15]. In addition to the momentum equations, transport equations for species mass fractions and specific enthalpy are solved fully coupled in order to ensure that internal feedback within the gas phase chemistry is inherently captured. For the present flame, a finite-rate chemistry (FRC) approach is used for gas-phase chemistry and sectional models for PAH chemistry and soot evolution. As an accurate description of the gas phase chemistry is crucial especially in soot modeling, a detailed reaction mechanism consisting of 43 species and 403 reactions is used [16, 17]. It has been developed for the combustion of small hydrocarbons including ethylene and describes the formation of soot precursors like benzene, toluene and acetylene. In total 84 transport equations are solved on a tetrahedral grid consisting of 7 million nodes, which guarantees a good resolution of the turbulent structures in the combustor. The diffusive fluxes consider differential diffusion for the molecular fluxes while the turbulent ones are calculated using constant turbulent Schmidt and Prandtl numbers of 0.4. The WALE model [20] is used for subgrid turbulence modeling and the ratio of turbulent to molecular viscosity is mostly less than 10 in the combustion chamber. Accordingly, a major part of the turbulence-chemistry interaction is directly resolved, and a minor fraction is modeled as subgrid fluctuations using an assumed PDF approach $[18,19]$. The latter uses a multivariate $\beta$-PDF for all chemical species, which makes no assumptions regarding premixed or non-premixed conditions (see Ref. [7] for more details). The spatial discretizations for momentum and species transport are of second order in order to avoid high numerical dissipation, and a second order Crank-Nicolson scheme is applied for temporal discretization. The averages and RMS values of velocity and temperature agree well with experimental values [7] indicating that the combustion process is predicted 
very well. The present work combines the results of experiment and LES for an improved understanding of flame dynamics, whereas no attempts for further assessment of LES results are made.

\section{Structure of flow field and flame}

\subsection{Average structure}

Averages of velocity field, soot distribution and $\mathrm{OH}$ chemiluminescence (OH-CL, integrated along line-ofsight) measured in previous studies $[3,5]$ are shown in Fig. 1. Upstream of the secondary air injectors, the flow field exhibits the typical features found in confined swirl flames, namely a conical jet of unburned gas and an inner recirculation zone (IRZ) where hot burned gas is transported towards the flame base and there supports ignition of the unburned gas. Strong velocity gradients are present in the inner shear layer (ISL) between the jet and the IRZ. The flow of secondary air injected at $y=80 \mathrm{~mm}$ partly contributes to the upstream flow in the IRZ and the other part mixes into the flow leaving the chamber through the exhaust tube. The OH-CL distribution shows that the major part of heat release takes place within the jet and ISL in the range of $10<y<30$ $\mathrm{mm}$. Soot is mainly present along the outer boundary of the IRZ with a maximum at the upstream end around $y=20 \mathrm{~mm}$, whereas no soot is found in the upper central part of the IRZ. Previous studies by Geigle et al. [5] showed that the depletion of soot in this region is related to the flow of secondary air into the IRZ and the associated presence of $\mathrm{OH}$. The time-resolved analysis in the next sections will further highlight the pivotal role of secondary air in the IRZ.

Measurements at other operating conditions indicate that the structure of flow field is representative also of flames with other values of pressure, $P$ and $\phi$, whereas the location and rates of soot formation then change significantly $[3,5,6]$. The study of transient dynamics of soot in the remainder of this work, however, is limited to the single operating condition specified above.

\subsection{Instantaneous structures}

Figure 2a shows an exemplary instantaneous combined OH-PLIF and stereo-PIV measurement. The distribution of $\mathrm{OH}$ reveals several distinct zones of zero, low, medium and high levels of $\mathrm{OH}$ that will be analyzed further below. The blue color depicts the PIV particle image that represents the intensity of light scattered by tracer particles or, if present, soot particles. The velocity field exhibits the conical jet and IRZ already seen in the average flow field, and a pronounced vortex in the
ISL. The latter is part of a 3D helical vortex core (PVC) that precesses with a frequency of $440 \mathrm{~Hz}$. Previous studies showed that the PVC enhances fuel-air mixing [21] and also affects the soot distribution [3].

As expected, the cold high-density unburned gas (UG) entering from the nozzle below is characterized by zero levels of $\mathrm{OH}$ and a high particle density. In all other zones the particle density is low indicating the presence of hot burned gas. This large region of hot gas, in turn, is composed of several clearly separated zones with different levels of $\mathrm{OH}$. At the boundaries of the UG, elongated zones of high $\mathrm{OH}$ concentration mark the presence of reaction zones where $\mathrm{OH}$ is formed. Furthermore two distinct zones without $\mathrm{OH}$ labeled as RBG (rich burned gas) are visible in the ISL to the left and right of the IRZ. To explain the absence of $\mathrm{OH}$ in these regions, the burned gas composition of ethylene-air mixtures at equivalence ratios $0.5<\phi<2.5$ (determined with GasEq [22] for $p=3$ bar) is plotted in Fig. 2b. It is seen that notable $\mathrm{OH}$ concentrations are present for $0.5<\phi<1.5$ whereas $\mathrm{OH}$ is depleted for $\phi>1.5$. Therefore these zones are considered as burned gas of fuel-rich ethylene-air mixtures of $\phi>1.5$. The occurence of such local fuel-rich mixtures is due to incomplete mixing of the flows of fuel and air from the injector with an average $\phi$ of 1.2. The hot gas in the IRZ, labeled as LBG (lean burned gas), exhibits medium levels of $\mathrm{OH}$ indicating relatively lean conditions resulting from admixture of secondary air.

Figures 2c-i show instantaneous distributions of species mole fractions and temperature of a selected LES snapshot with a remarkably similar spatial structure as the measurement in Fig. 2a. The shape and location of zones of UG, RBG, LBG and the vortex are almost identical for the calculated $\mathrm{OH}$ displayed in Fig. 2c. As expected from the admixture of secondary air, oxygen is present in the LBG but not in the RBG. The RBG contains major concentrations of $\mathrm{CO}$ and $\mathrm{H}_{2}$ resulting from decomposition and conversion of the excess fuel (see Fig. 2b) that are not found in the LBG (the regions of high values of $X_{\mathrm{CO}}$, which is not shown in Fig. 2, are almost identical to those of $X_{\mathrm{H} 2}$ ). Temperatures in the RBG range between $1900 \mathrm{~K}$ and $2100 \mathrm{~K}$ whereas $T \approx 1600 \mathrm{~K}$ in the LBG.

\subsection{Classification of reaction zones}

The LES results further reveal that two distinct types of reaction take place, for which representative examples are marked with red lines in Fig. 2c. The associated concentration profiles of major species plotted in Fig. $2 d$ show that premixed flame (PF) fronts are present at the boundaries of the UG. At the boundaries between 

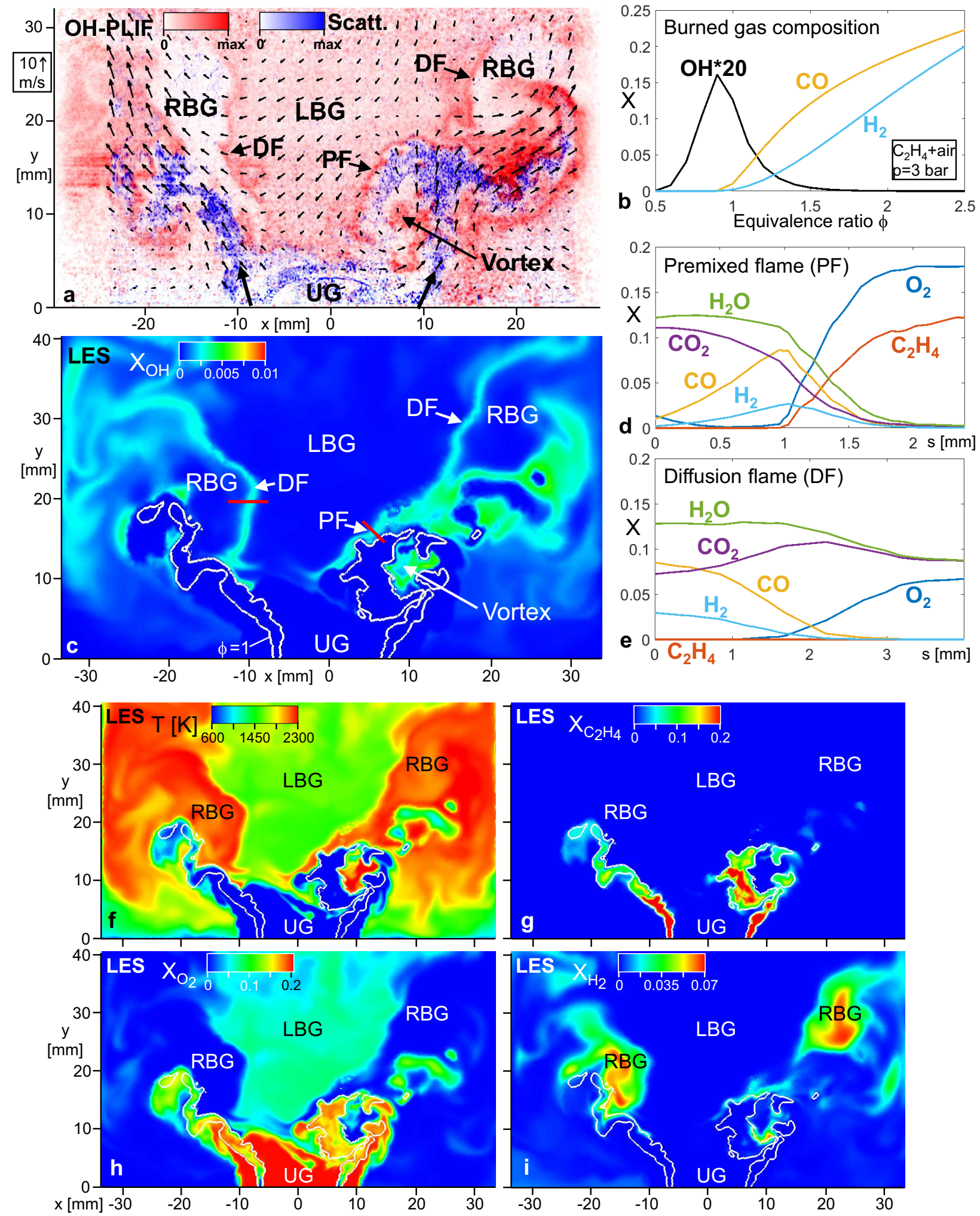

Figure 2: a) Measured instantanous distributions of $\mathrm{OH}$ (red), particle light scattering (blue) and velocity; b) calculated burned gas mole fractions of ethylene-air mixtures; c)-i) LES results for major species mole fractions and temperature. 

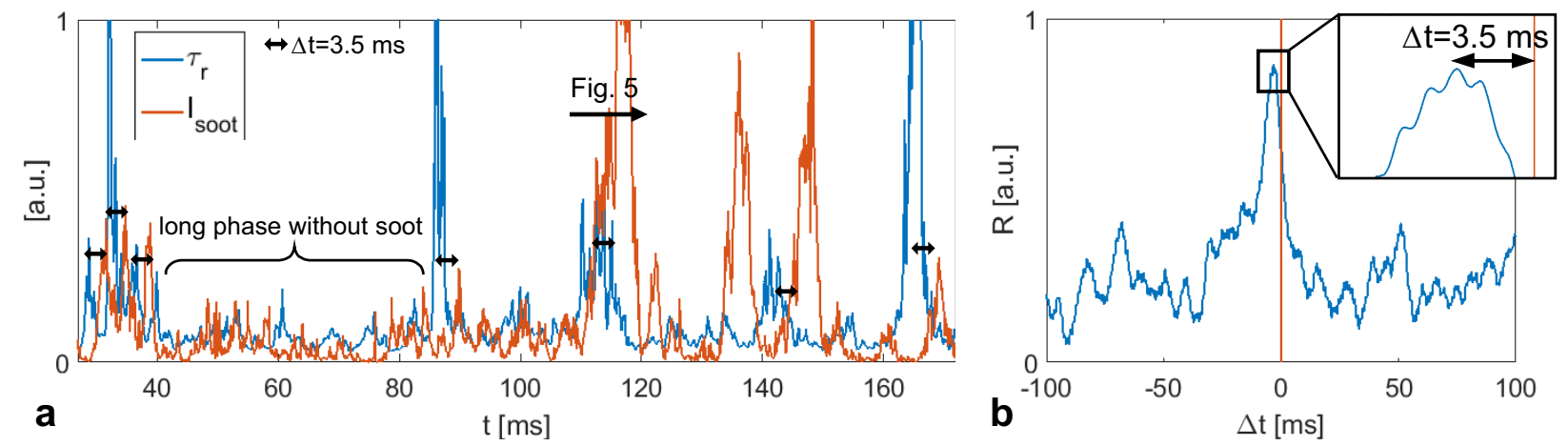

Figure 3: a) Time-series of soot intensity $I_{\text {soot }}$ and residence time $\tau_{\mathrm{r}}$ in the IRZ and b) cross-correlation $R$ of $I_{\text {soot }}$ and $\tau_{\mathrm{r}}$.
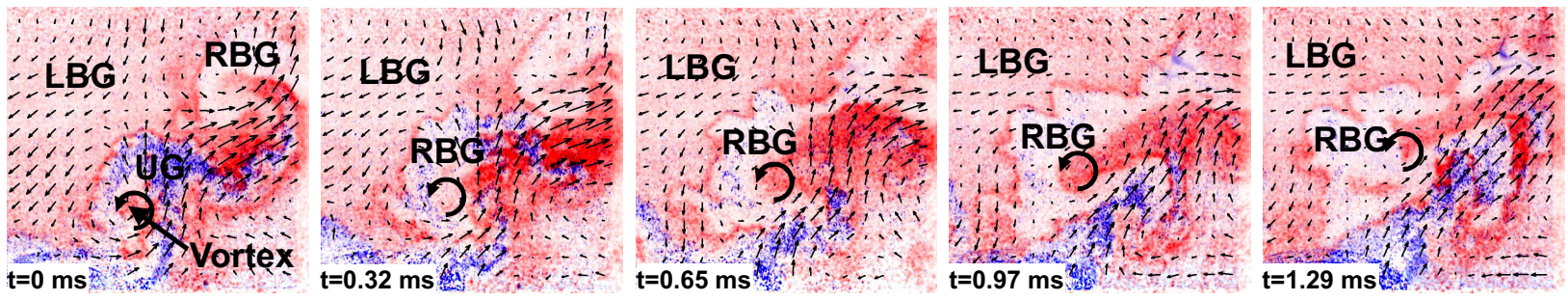

Figure 4: Time-series of PIV and OH-PLIF measurements showing formation and transport of RBG in the IRZ.

RBG and LBG, by contrast, diffusion flames (DF) are present. The corresponding profiles in Fig. 2e show that in the $\mathrm{DF}, \mathrm{CO}$ and $\mathrm{H}_{2}$ from the $\mathrm{RBG}$ are oxidized by $\mathrm{O}_{2}$ from the LBG. The good agreement of the locations of $\mathrm{PF}$ and DF reaction zones between OH-PLIF and LES demonstrates that the FRC model can well capture transitions between PF- and DF-type reactions.

\section{Dynamics of soot formation}

\subsection{Intermittency}

Based on the knowledge of the flame structure obtained in the previous section, the mechanisms of soot formation and depletion are now analyzed using the measured time-series. In the PIV images, the Mie scattering of PIV tracer particles results in clouds of separate dots representing individual particles, whereas the much smaller soot particles are imaged by Rayleigh scattering. The time-series in Fig. 5, which is discussed further in Sect. 4.3, shows that soot appears in the form of smooth filaments located mainly in the IRZ, whereas a high PIV particle density is mostly found in the dense unburned gas above the injector nozzle. In order to track the appearance of soot over time, the individual dots representing PIV tracer particles were removed from the
PIV images using a specifically developed image processing routine, such that only the smooth soot structures remain (if present). In Fig. 3a a section of the resulting time-series of the spatially integrated soot intensity $I_{\text {soot }}$ is plotted. It is observed that the occurence of soot is highly intermittent, with longer periods of absence and intermediate bursts of high intensity.

Another relevant quantity is the recirculation of LBG in the IRZ. First, the $\mathrm{OH}$ radicals present in the $\mathrm{LBG}$ can oxidize soot $[12,23,24]$, and second, the flow rate into the IRZ affects the residence time $\tau_{\mathrm{r}}$ of RBG within the IRZ, which must be sufficiently long to enable soot formation as argued below in Sect. 4.3. The flow of LBG into the IRZ is thus evaluated as the average of negative axial velocities at the upper boundary of the PIV domain $(y=31 \mathrm{~mm})$, denoted as $v_{\text {IRZ }}$. The value of $\tau_{\mathrm{r}}$ is then estimated as $\tau_{\mathrm{r}}=L / v_{\mathrm{IRZ}}$, where $L$ denotes a characteristic size of the IRZ. Since a precise determination of flow pathlines in a complex 3D swirling turbulent flow is not feasible from measurements in a $2 \mathrm{D}$ section, the estimates of $v_{\text {IRZ }}, L$ or $\tau_{\mathrm{r}}$ cannot be considered as accurate quantitative values. The discussion below will therefore only consider relative changes of $\tau_{\mathrm{r}}$, which seems reasonable to demonstrate the correlations between soot formation and fluctuating flow into the IRZ. 
The comparison of normalized $\tau_{\mathrm{r}}(t)$ and $I_{\text {soot }}(t)$ plotted in Fig. 3a shows that the periods of high soot intensity are often, yet not always, preceded by phases of long residence time, i.e., low velocity of recirculating LBG. Accordingly the cross-correlation $R$ between $\tau_{\mathrm{r}}(t)$ and $I_{\text {soot }}(t)$ plotted in Fig. $3 \mathrm{~b}$ exhibits a pronounced peak that quantifies this time-shift as $\Delta t=3.5 \mathrm{~ms}$. The clear correlation of $\tau_{\mathrm{r}}(t)$ and $I_{\text {soot }}(t)$ suggests that the formation of soot in the IRZ is goverened by the flow in the IRZ. The time-shift $\Delta t=3.5 \mathrm{~ms}$ is related to a combination of the convective time of burned gas in the IRZ and the chemical time for formation and oxidation of soot in the RBG, as elucidated below using image sequences from different phases of the process.

\subsection{Formation and transport of $R B G$}

Figure 4 shows the dynamics of flow field and flame during a phase where $\tau_{\mathrm{r}}$ is low and soot is absent. The time $t=0 \mathrm{~ms}$ corresponds to the measurement shown in Fig. 2a. It is seen that the UG around the vortex starts reacting at $t=0.32 \mathrm{~ms}$ and forms a pocket of RBG that continuously increases in size and then fills a significant part of the IRZ at $t=1.29 \mathrm{~ms}$. It is known that burned fuel-rich $(\phi \gtrsim 1.7)$ ethylene-air mixtures provide favorable conditions for soot formation [25]. On the other hand, presence of $\mathrm{OH}[12,23,24]$ or high levels of strain $[11,26]$ can inhibit soot formation. It is thus plausible that soot formation in the IRZ is suppressed when $\tau_{\mathrm{r}}$ is low, i.e. the velocity of OH-rich LBG into the IRZ is high as seen in Fig. 3.

\subsection{Transient formation and oxidation of soot}

Figure 5 presents an image sequence where an increase of $\tau_{\mathrm{r}}$ and a time-shifted increased soot formation take place (marked in Fig. 3a). At $t=110.3 \mathrm{~ms}$, an extended zone of RBG has formed in the left part of the IRZ, while the recirculation of LBG into the IRZ is very low. Subsequently the inflow of LBG remains low, and thus more and more RBG that is formed continuously in the ISL accumulates in the IRZ as seen at $t=113.2$ and $116.1 \mathrm{~ms}$. Soot starts forming under the favorable conditions in the RBG at $t=113.2$ and covers the major part of the IRZ at $t=116.1 \mathrm{~ms}$. At $t=118.1 \mathrm{~ms}$, recirculation increases and LBG enters the IRZ. Soot is depleted in the OH-rich LBG, whereas a major soot pocket is transported downstream and other soot filaments have moved towards the flame base. At $t=118.7$ and $120.3 \mathrm{~ms}$, the recirculation of LBG remains high, and thus the IRZ is more and more filled with OH-rich LBG such that soot formation is inhibited. In summary, both the time-series in Fig. 3 and the image sequence in Fig. 5 clearly indicate that the intermittent formation and oxidation of

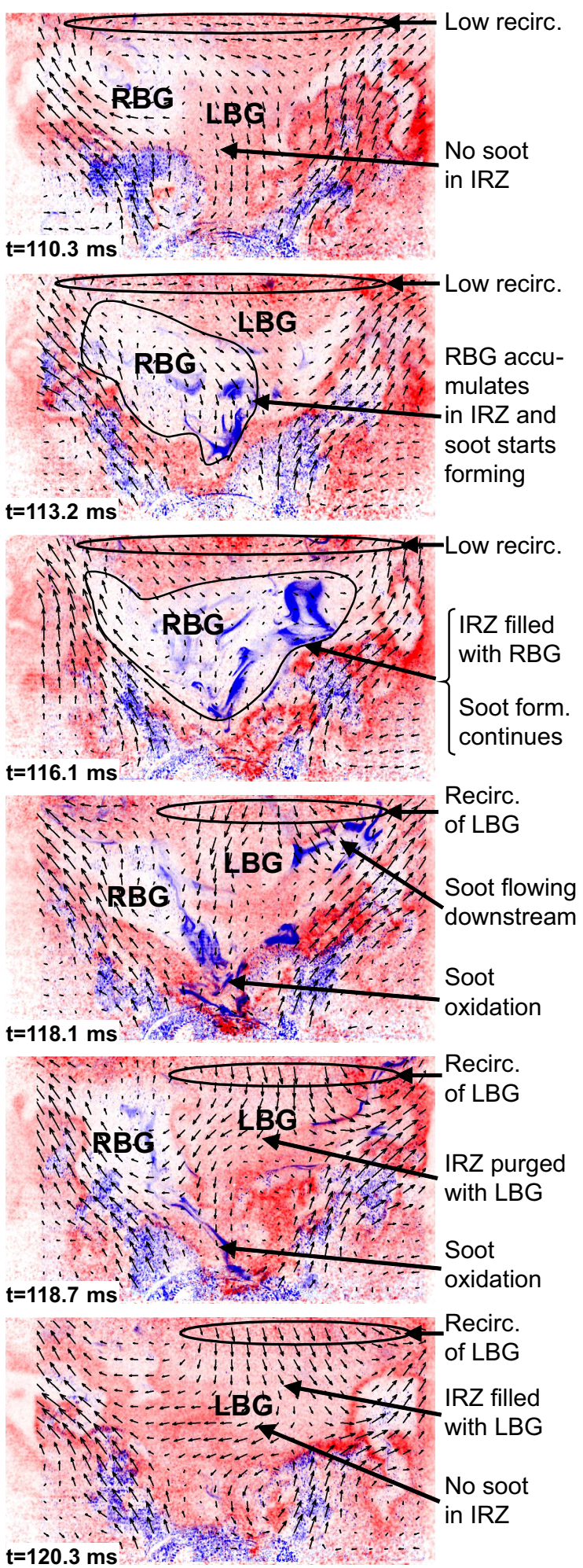

Figure 5: Time-series of PIV and OH-PLIF measurements showing the evolution of soot formation, transport and oxidation in the IRZ. 
soot in the IRZ are controlled by the intermittent flow in the IRZ. Both the PIV measurements by Geigle et al. [3] and the LES of Grader et al. [7] revealed a significant flapping of the jets of secondary air, which might be the source of the flow intermittence.

\section{Conclusions}

The highly transient and history-dependent dynamics of soot studied in this work demonstrate the importance of time-resolved laser-based measurements for an advanced understanding of soot formation in aero-engine combustors. The results further highlight the essential role of additional information from detailed LES for a proper interpretation of OH-PLIF measurements in complex turbulent flames. The combined analysis showed that the evolution of soot is governed by an unsteady interplay of distinct pockets of burned gas with either relatively rich or relatively lean composition. The time-resolved complementary measurements of velocity field and flame structure allowed tracking of both the burned gas pockets and soot filaments. It was seen that soot generally forms in the rich pockets if their residence time in the IRZ is sufficient, whereas oxidation occurs in the lean zones carrying $\mathrm{OH}$. Correlating the dynamics of flow field and soot indicated that the intermittency of soot is driven by the intermittent flow of lean burned gas in the IRZ that might in turn be driven by flapping of jets of secondary air further downstream. This suggests that the soot formation might be reduced by a proper adjustment of secondary air injection aiming at a sufficient and more constant recirculation of LBG.

It was further revealed that both premixed and diffusion-type flame fronts are present in the flame, and the locations of these flame fronts were found to be similar in selected instantaneous snapshots of OH-PLIF and LES. A good agreement of measurement and LES was also found in terms of average and RMS of velocities and temperatures, which further indicates that flow field and gas-phase reactions are well predicted by the LES. The experimental insights into the transient mechanisms of soot formation and oxidation, on the other hand, may provide valuable input for LES soot models where deviations from measurements are generally larger.

\section{Acknowledgments}

Funding from the EU within the project SOPRANO (Grant No. 690724) and the Air Force Office of Scientific Research under Award No. FA9550-16-1-0044 is gratefully acknowledged. C. Carter is supported by the Air Force Windows on the World program.

\section{References}

[1] M. B. Colket, in: T. Lieuwen, V. Yang (Eds.), Gas Turbine Emissions, Cambridge University Press, 2013.

[2] H. Wang, Proc. Combust. Inst. 33 (2011) 41-67.

[3] K. P. Geigle, R. Hadef, M. Stöhr, W. Meier, Proc. Combust. Inst. 36 (2017) 3917-3924.

[4] M. Mueller, H. Pitsch, Phys. Fluids 25 (2013) 110812.

[5] K. P. Geigle, M. Köhler, W. O'Loughlin, W. Meier, Proc. Combust. Inst. 35 (2015) 3373-3380.

[6] K. P. Geigle, W. O’Loughlin, R. Hadef, W. Meier, Appl. Phys. B 119 (2015) 717-730.

[7] M. Grader, C. Eberle, P. Gerlinger, M. Aigner, Proc. ASME Turbo Expo (2018) GT2018-75254.

[8] A. Wick, F. Priesack, H. Pitsch, Proc. ASME Turbo Expo (2017) GT2017-63293.

[9] B. Franzelli, E. Riber, B. Cuenot, M. Ihme, ASME Turbo Expo (2015) GT2015-43630.

[10] M. Köhler, I. Boxx, K. P. Geigle, W. Meier, Appl. Phys. B 103 (2011) 271-279.

[11] V. Narayanaswamy, N. T. Clemens, Proc. Combust. Inst. 34 (2013) 1455-1463.

[12] B. Franzelli, P. Scouflaire, S. Candel, Proc. Combust. Inst. 35 (2015) 1921-1929.

[13] J. B. Michael, P. Venkateswaran, C. R. Shaddix, T. R. Meyer, Appl. Opt. 54 (2015) 3331-3344.

[14] B. Wieneke, Meas. Sci. Technol. 26 (2015) 074002.

[15] J.-M. Lourier, M. Stöhr, B. Noll, S. Werner, A. Fiolitakis, Combust. Flame 183 (2017) 343-357.

[16] N. A. Slavinskaya, U. Riedel, S. B. Dworkin, M. J. Thomson, Combust. Flame 159 (2012) 979-995.

[17] T. Blacha, M. Di Domenico, P. Gerlinger, M. Aigner, Combust. Flame 159 (2012) 181-193.

[18] S. Girimaji, Combust. Sci. Technol. 78 (1991) 177-196.

[19] P. Gerlinger, Combust. Sci. Technol. 175 (2003) 841-872.

[20] F. Nicoud, F. Ducros, Flow Turbul. Combust. 62 (1999) 183200.

[21] M. Stöhr, C. Arndt, W. Meier, Proc. Combust. Inst. 35 (2015) 3327-3335.

[22] C. Morley, Gaseq v0.79, 2005 http://www.gaseq.co.uk.

[23] S.-Y. Lee, S. R. Turns, R. J. Santoro, Combust. Flame 156 (2009) 2264-2275.

[24] H. Ghiassi, D. Lignell, J. S. Lighty, Energy Fuels 31 (2017) 2236-2245.

[25] P. Markatou, H. Wang, M. Frenklach, Combust. Flame 93 (1993) 467-482.

[26] M. E. Mueller, Q. N. Chan, N. H.Qamar, B. B. Dally, H. Pitsch, Z. Alwahabi, G. Nathan, Combust. Flame 160 (2013) 12981309. 